\title{
Analysis of the human risk factor which affects the reliability and safety of machinery
}

\author{
G. Hotta ${ }^{1}$, D. Ishibashi ${ }^{1}$, Y. Ohbuchi ${ }^{2}$ \& H. Sakamoto ${ }^{3}$ \\ ${ }^{I}$ Department of Mechanical Engineering, \\ Ariake National College of Technology, Japan \\ ${ }^{2}$ Creative Engineering and Design Education Center, \\ Kumamoto University, Japan \\ ${ }^{3}$ Graduate School of Science and Technology, \\ Kumamoto University, Japan
}

\begin{abstract}
It is an important matter to decrease the mean time to repair (MTTR) in order to improve the reliability of machinery. When the factory workers repair as non routine operations, there is no operation manual. Therefore, the repairing quality depends on the maintenance engineer's skill or the operator's skill. However, in the non-routine operations, the correlation of the maintenance engineer's experience and the decrease of MTTR does not necessarily exist. This is an important problem in optimum maintenance planning. So, an analysis of the influence of the human factor on the reliability of machinery is desired. In this research, we make an action model for coping with the failures of maintenance engineers, and analyze the human factor which affects the reliability of machinery. In addition, we consider the improvement of the reliability of machinery by reducing the MTTR.
\end{abstract}

Keywords: MTTR, infrequent operation, human factor, risk-taking situations, psychological tests, risk reduction.

\section{Introduction}

Production facilities keep their reliability by check and maintenance. The routine check and maintenance procedure is generally carried out based on a check manual. In the case of the breakdown of newly installed machinery, the repair manual sometimes cannot be found in the operation text. In the initial failure 
period of the Bath Tub Curve in particular, this tendency is notable. When there is mention that "it is easy to repair" as a measure of the reliability in the mechanical equipment, it is indispensable to reduce the MTTR by the immediate restoration of a breakdown at a production site. As there is no repair manual in non-routine work, the repair operation is completely the responsibility of the maintenance and operating engineer. This operation largely depends on the engineer's skill and experience [1]. However, in non-routine operations, the correlation of the maintenance engineer's experience and the decrease of MTTR does not necessarily exist [2]. This is an important problem in optimum maintenance planning. In this research, an analysis of the influence of the human factor on the reliability of machinery was carried out and the reliability improvement of machinery by reducing the MTTR and the repair time was discussed.

\section{Action model of the maintenance engineer during the breakdown}

\subsection{Conventional study about reliability and human risk}

Past research on the influence of the human factor on the reliability of machinery is as follows: 1) the quality engineering analysis about the reliability of software in the design review process [3]; 2) the occurrence pattern of human error in the marine vessel's engine problems [4]; 3) the system's reliability analysis on the relation between human behavior and machinery breakdown, etc. In the conventional study, human error is defined as a factor of the reliability in a model of a manually operated machine and its countermeasure is considered. In this research, we paid attention to the individual personality and behavioral characteristics of the maintenance engineer and not to human error as the risk factor affecting the reliability of the machine. These are pre-behavior, current behavior and post-behavior at time of the breakdown.

\subsection{The influence of a personal behavior model on reliability}

According to a Japanese preservation society, the operating engineer's behavior at time of breakdown was classified into the following three parts: 1) planning, 2) action and 3) evaluation [6]. These are termed pre-behavior, current behavior and post-behavior at breakdown. Evaluation is the report of the cause of breakdown and the repair treatment, and the collection of the information for the prevention of recurrence. As the reliability of a mechanical system is decided by the combination of these three behavior items, we define each behavior element of a maintenance engineer as $K 1, K 2$ and $K 3$. A machine causes $N$ time breakdown during a fixed period, and we assume that different maintenance engineers can handle the breakdown. The set of the choices for repair behavior were given to maintenance engineers of $N$ person. We assign the number to each maintenance engineer of $N$ person and consider the next assembly. 


$$
I=\{1,2,3, \cdots \cdots, N\}
$$

The behavior choices $(\mathrm{a}, \mathrm{b}, \cdot \cdot \cdot)$ which a maintenance engineer decides, exists in each behavior element $K n(n=1,2,3)$. So the next system is formed.

$$
K_{n}^{I}=\left\{a_{n}^{I}, \quad b^{I}{ }_{n}, c^{I}{ }_{n} \cdots \cdot\right\} \quad(n=1,2,3 \cdot \cdots)
$$

As a result, the repair time at each behavior element is determined when a maintenance engineer selects the repair behavior. For example, when he selects the behavior of $K I$ (plan) stage, the assembly $T_{K I}$ of all repairing time is represented as the following system.

$$
T_{K I}^{I}=\left\{t_{K I}{ }^{1}, t_{K I}^{2}, t_{K I}^{3} \cdot \cdots\right\}
$$

When we assume the assembly which collects the repair time as the result of executing all of the three behavior elements to be $R^{I}$.

$$
\overline{R^{l}}=\left\{\left(T_{K 1}^{1}+T_{K 2}^{1}+T_{K 3}{ }^{1}\right),\left(T_{K 1}^{2}+T_{K 2}^{2}+T_{K 3}^{2}\right), \cdots \cdot\right\}
$$

Because selecting from a number of repair method candidates becomes a decision making problem, the maintenance engineer's behavior affected on $R^{I}$ can be considered in the cases of the following two types (4) and (5).

$$
\begin{gathered}
u\left(R_{\text {work }}-\overline{R^{I}}\right)>p \cdot u\left(R_{\text {work }}-R_{\text {loss }}\right)+(1-p) u \cdot R_{\text {work }} \\
p \cdot u\left(R_{\text {work }}+R_{\text {loss }}-\overline{R^{I}}\right)+(1-p) u\left(R_{\text {work }}-\overline{R^{I}}\right)>u \cdot R_{\text {work }}
\end{gathered}
$$

Here, $\overline{R^{l}}$ is the average maintenance time, $R_{\text {loss }}^{I}$ is lost time by judgment error, $R_{\text {work }}^{I}$ is the standard working hour, and the probability of judgment error is $p$.

Equation (4) shows the action of the risk avoidance and Equation (5) shows the action of risk orientation. In this study, we calculate the utility function $u$ shown in Eqs. (4) and (5), which is obtained by experiment and we examine whether the behavior of a maintenance engineer is risk avoidance type or risk orientation type. However, as there is a report that even the person of the risk evasion tendency has a tendency to become the risk orientation type in group [7], we also investigated the change in the action pattern in the group behavior.

\subsection{Risk behavior model of the maintenance engineer}

The professional attribute and individuality of the maintenance engineer are generally different. So assembly $R_{I}$ in repairing time about Eq. (3) has too many elements. Therefore, the searching of utility function $u$ which satisfies equation (4) is not practicable. Here, the professional attribute means his experience and skill and the individuality means inherent characteristics like his strong and weak points and his reactive response, etc. So we simplify the behavior model and make the following suppositions for checking the utility function by experiment easily: i) the maintenance engineer's professional 
attribute is uniform; ii) the maintenance engineer's professional individuality depends on preference strength between 3 elements $K_{1}, K_{2}$ and $K_{3}$ of a behavior model, because the professional attribute and individuality of the maintenance engineer are generally different for every individual; iii) the operation is non routine works. To avoid the complexity, supposition i) was installed according to the study of Hirose et al. [8]. The preference strength [9] of ii) means the different priority order of three behavior patterns by strong and weak points.

Figure 1 shows the analysis of a "close call" report by a chemical company in Fukuoka Prefecture, Japan. The first action of the employee after touching the breakdown valves is indicated. From this figure, it is found that the employee takes a different action respectively, and these actions are classified into the following three behavior patterns; the guess, the action and the information gathering. From the response behavior for the breakdowns, it is guessed that there are the different behavior patterns by each employee.

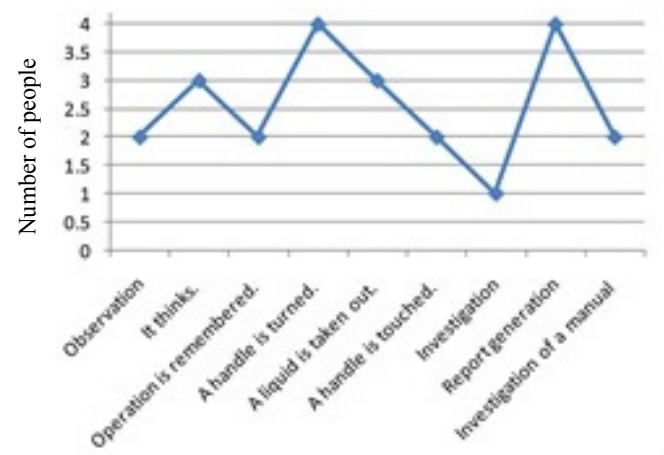

Figure 1: Action after touching the broken valve for the first time.

The supposition iii) is the necessary condition to fill the supposition ii). There is each behavior pattern of the maintenance engineer by the preference order of three action elements from the above mentioned supposition. The effect of the behavior pattern on the repair time is examined, and the estimation of the utility function $u$ become possible.

\section{Behavior pattern check of the maintenance engineer}

\subsection{Presumption of behavioral pattern by the maintenance engineer's preference}

In order to study the behavior patterns that structure the preference relation among the behavior elements, the following survey questionnaire, mentioned in this paper as experiment (1), was carried out on 63 driver license holder $3^{\text {rd }}$ grade students of the Department of Mechanical Engineering of Sojo University. 
"Please select at least two of the following (1), (2) or (3) items. If you think you have merits for one item, indicate the corresponding number, and if not, write a 0 . (1) I can predict the risk to some extent; (2) I can avoid dangers even when sudden; (3) when informed by another person of a dangerous experience, I use this safety information as a new lesson."

From the questionnaire's results, 11 kinds of behavior patterns have been distinguished by preference relation and named respectively I-type-XI-type. The number of persons corresponding to each type is given in Table 1 in field "number of person (1)" and the preference relations are represented by mathematical symbols.

Table 1: Difference of the repair time by an action pattern.

\begin{tabular}{|c|c|c|c|c|}
\hline \multirow[t]{2}{*}{ Type } & \multirow[t]{2}{*}{$\begin{array}{l}\text { Preference } \\
\text { relation }\end{array}$} & \multicolumn{3}{|c|}{$\begin{array}{l}\text { The number of People } \\
{[\%]}\end{array}$} \\
\hline & & (1) & (2) & (3) \\
\hline I & (1) $\sim$ (2) $\sim$ (3) & 10 & 10 & 11 \\
\hline II & (1) $\succ$ (2) $\succ$ (3) & 25 & \multirow{2}{*}{13} & 26 \\
\hline III & (2) $\succ$ (1) $\succ$ (3) & 9 & & 16 \\
\hline IV & (2) $\succ$ (3) $\succ$ (1) & 13 & \multirow{2}{*}{18} & 0 \\
\hline V & (3) $\succ(2)^{\succ}$ (1) & 5 & & 0 \\
\hline VI & (1) $\succ$ (3) $\succ$ (2) & 19 & \multirow{2}{*}{5} & 21 \\
\hline VII & (3) $\succ$ (1) $\succ$ (2) & 0 & & 0 \\
\hline VIII & (1) $\succ(2) \sim(3)$ & 0 & 0 & 0 \\
\hline IX & (2) $\succ$ (1) (3) & 13 & 26 & 21 \\
\hline $\mathrm{X}$ & (3) $>$ (1) (2) & 0 & 3 & 0 \\
\hline $\mathrm{XI}$ & (1) (2) (3) & 6 & 25 & 5 \\
\hline
\end{tabular}

In addition, when only two items were selected, it is clear that the remaining item has a very low preference order and therefore a preference relation is used among the three. And when all the items are equivalent (all set to 0 or blank) they are assumed to be indiscriminate.

The preference relations obtained from the questionnaire of experiment (1) are displayed in Figure 2 overlaying the number of person. The preference relations obtained from the questionnaire of experiment (1) are displayed in Figure 2 overlaying the number of people. By comparing the numbers of people of experiment (1) it can be seen that there are more less behaviors of types XI and IV (but also V). Since type XI corresponds to an unconscious behavior and type IV denotes inattention they are considered to be failures. 


\subsection{Comparison of the behavior pattern and a member of society}

From experiment (1) we could distinguish 11 types of behavior patterns. Here we check if such patterns also apply to factory workers using the following case method. The case method was first applied on 36 students of the Mechanical Engineering Department of Ariake National College of Technology. The case method consists of: (1) plan before operation (prevision of risks); (2) safety measures during the operation (risk aversion); and (3) report of the experienced risks (transmission of risk knowledge). The resulting priority order was compared with the one of experiment (1) to see to what extent they correspond.

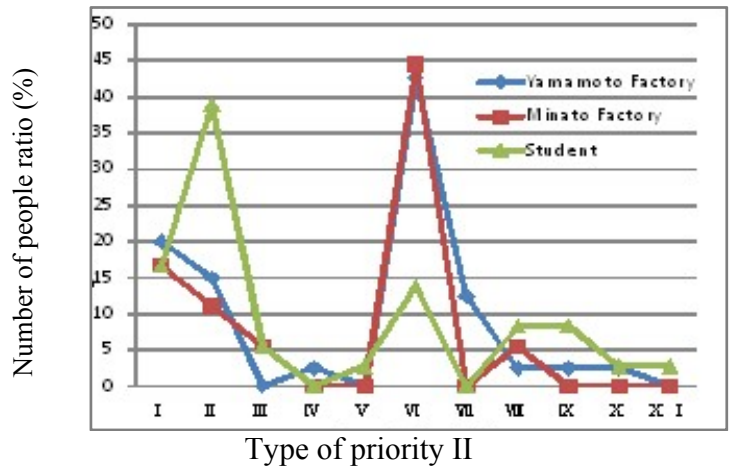

Figure 2: Comparison of a worker and a student in a case method result.

Then, we did the same with 40 employees of a factory of Yamamoto Co. (which produces cans, and includes large machinery), and 18 employees of Ltd. Harbor handicraft (which includes small machinery). Those two enterprises have been chosen because both because the daily manufacturing work includes the production of a large part of low-volume non-standard products. The results of these new experiments are given in Figure 2. This figures first shows that an enterprise worker is roughly equivalent to a student, which validates using a student as a model of an enterprise worker performing non-regular tasks. However, the characteristics of response to danger prove to be different between students and professionals. As seen in Figure 2, the students chose in majority behavior type II when facing a danger whereas for both enterprises the workers chose behavior type IV that give priority to the transmission of information about the experienced risk over the prevision of risk. In other words, communication among the partners is emphasized in the professional world.

\subsection{Comparison of the action patterns in a student and a member of society}

During a survey, the employee tends to answer taking into account their manager. Hence, in order to ascertain the authenticity of the survey results, we imposed the machine operations used for the experiments with the 40 employees of the Yamamoto Corp. factory. Figure 3 shows the experimental apparatus: a hand 
stamper used for printing on paper. The subject prints "-" on paper with a stamp. " $\Lambda$ " is printed by the paper already. He can print the character as $C$ according to the " $\mathrm{A}$ ". We requested that he did this using his non dominant hand.

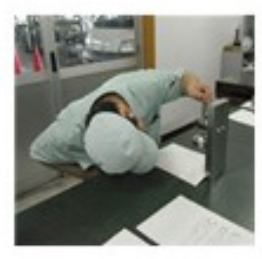

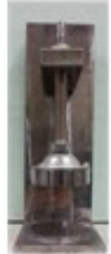

(a)

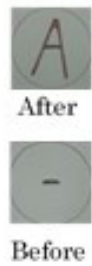

(b)

Figure 3: $\quad$ Experimental apparatus: (a) manual stamper; (b) printer character.

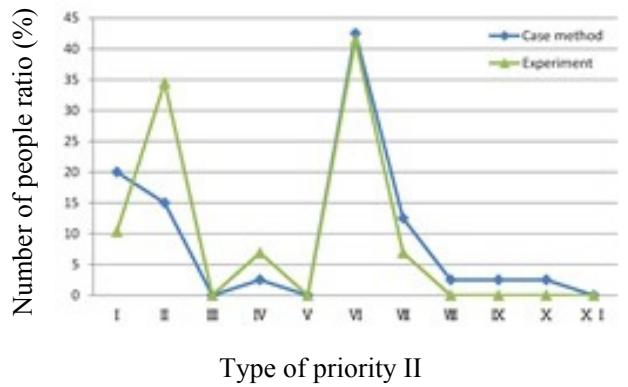

Figure 4: Comparison with the results so far and the questionnaire after the experiment.

The characteristics of the task were obtained through the observation the operation and the questionnaire filled out after work. The questionnaire consisted of giving the preference order among (1) before the task, the planning of the operations to apply; (2) during the task, the avoidance of failure; (3) after the task, the transmission of information to the next worker. The results of this experiment are shown in Figure 4. This figure also shows the results of the previous experiments, and shows that in both cases, type VI was the most selected behavior, the following one being type II. The behavior characteristics of risk presumption and risk avoidance were both significant in the case of nonregular work when the worker acted as an individual, but the prevention of risk proved to be stronger when the worker acted as an employee. When a risk was known to be possible, types VII and X, those who postpone risk avoidance, formed about $50 \%$ of the prevision of risk behaviors. 


\section{Change in action pattern in grouping}

\subsection{Change in individual action in a group}

Nowadays, work organizations become more and more collective, so the number of interactions within workplace groups is increasing.

Thus, the employees in industrial fields are acting as members of an organized group rather than as individuals, and their management too requires to be performed collectively rather than independently. Hence we studied the change in behavior patterns depending on the number of individuals in a group for the cases of experiment (2). For that purpose, we made 5 groups of 3 manufacturing staff of the studied Yamamoto Corp. Using the same basic framework as for Figure 3, three different print patterns were to be performed with 3 manual stampers. The same experiment was also done with 10 different persons individually to be compared with the result of the groups afterwards. Each individual worker had to operate 2 or more manual stampers and an award was to be given if 2 or more stamps had been printed out beautifully. The other rules ( 3 and 4 items) were similar.

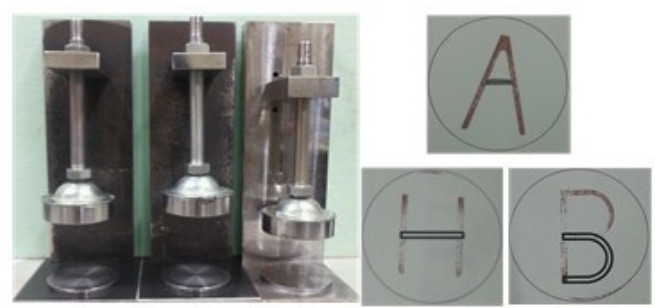

Figure 5: $\quad$ Experimental apparatus: (a) manual stamper; (b) printer character.

The questionnaire to fill out after work as an addition to the 3 and 4 items, included (1) the obtained awards, (2) the operation during the experiments and (3) which of aspect of the effort about quality insurance was the staff most aware of.

\subsection{Experimental results}

The result of the group work experiment is given in Figure 6. In the figure it can be seen that individual workers favor behavior type II whereas group workers prefer behavior type IV, which confirms that the tendency toward risk presumption increases in collective work. Furthermore, Figures 7 gives the results of the questionnaire about what is the worker aware of during the experiments and shows that numerous individual workers were aware of the impact of the effort on the quality insurance, whereas the worker in-group focused more in obtaining the award rather than the quality insurance, which was the original objective of working in a group. Consequently, when an individual 
worker is put into a group, there is an increase of the risk presumption and a decrease of the motivation which is a dangerous tendency.

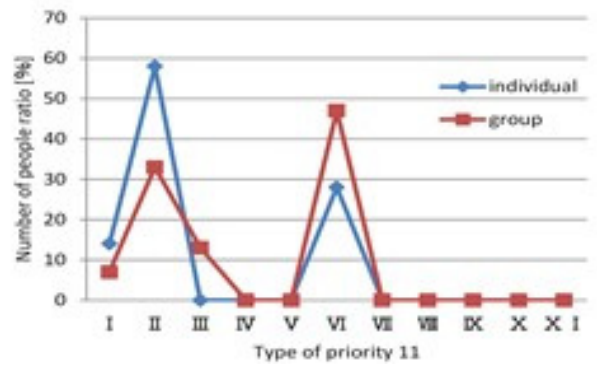

Figure 6: Questionnaire results after the Figure 7: experiment.

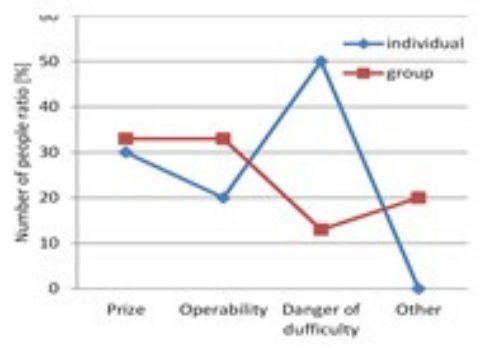

Consciousness

during experiment.

\section{Evasion of risk orientation by grouping}

Experiments presented in the previous section show that when performing nonregular tasks, the behavior pattern of each individual varies a lot, and that there is a tendency toward risk-oriented behavior when working in a group. It is therefore necessary to analyze quantitatively the change in behavior pattern for an individual when working in a group in order to understand it, and improve the prevention of labor accidents. For that purpose, a new experiment was performed. 25 employees of the Yamamoto Corp. factory where divided into 6 groups of 3 people, and the remaining 7 were set to work alone. The task considered for the experiments (as illustrated in Figure 8), consisted of putting beans onto a plate using chopsticks within one minute. Each single bean put onto the plate scored one point, but when two beans were put at one time the score was doubled to four points. During the experiment, each member of a group was set to work separately from the others so that he could not see the skills of his co-workers. With this experiment, each subject had to choose before a safe behavior (taking

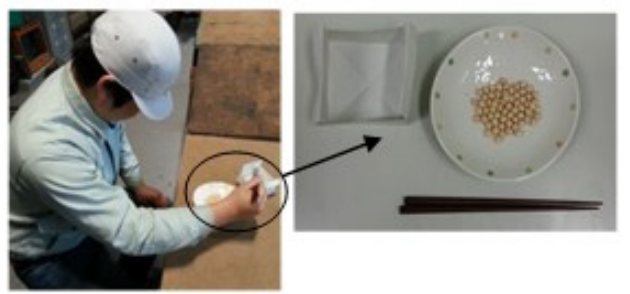

Figure 8: During the experiment. 
beans one by one) and a risk-taking behavior (taking beans two by two). When choosing the safe behavior the risk of failure is low, but the reward is low too. By contrast, when choosing the risk-taking behavior, a large score will be achieved if performing well, but it is possible to lose a lot of time due to numerous failures. During the experiments, the number of people choosing the risk-taking behavior were checked every ten seconds.

In addition, a questionnaire was given to the workers that asked them to indicate the behavior they choose for every time period of ten seconds. Figure 9 gives the results of this experiment. As seen in the figure, the employees that were not in a group tend to adopt a safe behavior at the beginning and then switch to a risk-taking behavior from the second half of the period, whereas the opposite is true for the in-group employees. Also, while individuals and group workers both chose to some extent a safe behavior, there is a higher probability for the group workers to be aware that they are following a risk-taking behavior. When comparing Figures 9(a) and 9(b), which represents, respectively, the observations result and the questionnaire results, it can be seen that the employees tend to think they are adopting a safe behavior while actually following a risk-taking behavior. It can also be seen from the figure that, even when aware of their behavior, in-group employees tend more toward a risktaking behavior than individual employees.

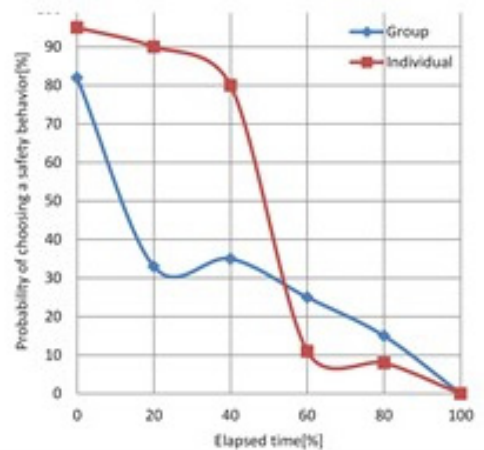

(a)

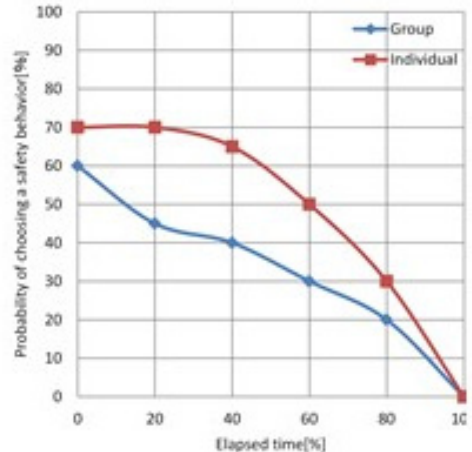

(b)

Figure 9: Experimental results: (a) observations during the experiment; (b) questionnaire results after the experiment.

\section{Conclusion}

In the reliability improvement of the mechanical equipment, it is necessary to decrease the entire MTTR by the shortening of one repair time. MTTR depend on a human factor like the preparation beforehand and the capability of the repair, etc. So, we proposed a concrete model of a human factor in this study, and the verification experiment was carried out. Here, as the model, the utility function 
based on the preference choice of the means was used. The preference alternatives consist of three action elements of plan, action and evaluation, which actions are the behaviour of the maintenance engineer who encountered the breakdown. How the restoration time changed by each maintenance engineer's priority choice was examined by the experiment. In three action elements, the individual difference for priority choice of behavior was recognized in a student. First of all, these three action elements were classified into 11 kinds of action patterns by the priority order. Next, the student was made to experience a mock breakdown, and 11 kinds of action patterns changed by grouping was examined. As a result, the risk orientation behavior by grouping increases, and the decrease of MTTR is obstructed. Then, the maintenance engineer has to receive the education to be conscious enough of the risk orientation before the maintenance engineer takes the action in order to improve the reliability of the mechanical equipment by decreasing MTTR. As mentioned above, a quantitative understanding of the correlation of the risk orientation of the maintenance engineer and the decrease obstruction of MTTR is important. An additional verification is scheduled in order to obtain the correlation by an additional experiment in the company in the future.

\section{References}

[1] H. Sugimoto, "Maintenance Work and Safety for Production Machine", Reliability Engineering Association of Japan, Vol. 25, No. 7, pp. 690-694 (2003).

[2] F. Baba, "Research on the stable equipment maintenance activities based on the PDCA cycle - The information link and division by class of a production activity as the foundation -", Yokohama International Society Study Research, Vol. 2, No. 2, pp. 210-211 (2012).

[3] K. Esaki, S. Yamada, M. Takahashi, K. Ihara, "A Quality Engineering Approach to Human Factors affecting Software Reliability in Design Process", The Journal of the Institute of Electronics, Information and Communication Engineers Vol. J83-A, No. 7, pp. 875-882 (2000).

[4] M. Najamura "Analysis of Human Errors in Marine Engine Accidents", Research Reports of the Yuge National College of Maritime Technology Vol. 34, pp. 14-19 (2012).

[5] T. Matuoka, "System reliability analysis in consideration of human action and an equipment failure - Approach by the GO-FLOW technique-", http://www.mech.utsunomiya-u.ac.jp/safety/gf_mmi.htm (2013).

[6] Japan Society Maintenology, "Examination about a preservation program and an inspection", The Data of Investigative Commission about the State of the 20th Inspection, pp. 6-11 (2006).

[7] I. Atunobu, "Theory of decision making”, Kyoritu Shuppan Co. Ltd, pp. 4-7 (1983).

[8] F. Hirose, D. Takeda, J. Fujimoto, “A Study on Measurement of Individual Hazard Perception (Part 3) - Development of Measurement Method of 
298 Safety and Security Engineering VI

Individual Hazard Perception -”, Report of research: Y10012 of Central Research Institute of Electric Power Industry, pp. 1-4 (2011).

[9] S. Terano, "A Guide to System Engineering", Kyoritu Shuppan Co. Ltd, pp. 168-172 (1996). 